\title{
Continuing research after training: perspectives from the IUGA fellows session 2021
}

\author{
Ankita Gupta ${ }^{1}$ \\ Published online: 19 January 2022 \\ (c) The International Urogynecological Association 2022
}

Urogynecology has undergone an explosion in scientific inquiry over the past 3 decades $[1,2]$. The requirement for 12 months of research in a 36-month fellowship program in the United States (US) and for a published thesis at many other training institutions around the world have supported these efforts. While research activity at training sites has increased, the transition from fellowship to clinical practice can be jarring for those wishing to incorporate research as part of their career after graduation. Of the 248 fellows who graduated between 2011 and 2020 in the US, 66\% currently hold no academic or faculty appointment while $30 \%$ hold junior faculty positions [3]. Fulfilling research potential is challenging for urogynecologists who enter non-academic roles without access to research funding or protected time. With this in mind, the Fellows, Trainees and Early Career Professionals Committee of the International Urogynecological Association (IUGA) put together a panel at the fellows' session held during the 2021 IUGA annual meeting to discuss resources needed for continued research after training.

Research mentorship was a key theme that emerged from this discussion. Early career faculty should seek out research mentors but also seek opportunities to act as research mentors for trainees. Helping medical students and residents develop and complete their research projects can advance career goals and hone research acumen for junior faculty. Identification of research mentors can greatly help early career faculty with resources and peer mentorship. For those without access to research mentors at their own institution, organizations such as IUGA should play a central role in connecting members with mentors with similar interests and greater experience.

Ankita Gupta

ankita.gupta@louisville.edu

1 Division of Female Pelvic Medicine \& Reconstructive Surgery, University of Louisville Health, 1900 Bluegrass Avenue, Louisville, KY 40215, USA
Research collaboration, another theme that emerged at the meeting, is an increasingly important aspect of research. Research collaborations can take many forms and can be intra-institutional, national, or international. Collaborations can be formed with other urogynecologists, physicians in other specialties, allied medical personnel such as physical therapists, and/or scientists working within women's health. Again, IUGA, as a melting pot for individuals from varied professions who have an interest in urogynecology, should play an active role in promoting research collaboration. All panelists expressed the importance of predetermining research roles, authorship criteria, and authorship order for these collaborations. This prevents disagreements at later stages, highlights short- and long-term goals, and sets clear expectations among team members leading to a fruitful process.

Partnerships with industry may facilitate early career professionals to receive financial and research support. Navigating these relationships can be tricky, and early stage investigators should learn from more experienced urogynecologists, if available. It is important to clarify the role of industry sponsors: funding only, patient recruitment, product acquisition, statistical support, etc., prior to entering such a collaboration.

As clinical specialists, urogynecologists have many demands on their time. For those without protected time, scholarly activities will most likely be completed after clinical hours. Identifying clinical questions and projects that harmonize with necessary clinical activities is key to maximizing research potential. Open discussions with employers may help new faculty identify sources of research funding and the value that scholarly activity brings institutions. Recent graduates should be aware of promotion requirements at their institution. Some academic centers do not count "middle" author publications toward promotions, and awareness of this may allow junior faculty to distribute their time appropriately. 
Despite potential challenges, research after FPMRS training is a worthy endeavor that can be undertaken by urogynecologists in clinically busy private practice. Collaboration and mentorship are key and can take various forms including accessing existing projects and initiating new research through organizations such as IUGA.

Acknowledgements I would like to thank our panelists, Drs. Rufus Cartwright (UK), Pattaya Hengrasmee (Thailand), and Javier PizarroBerdichevsky (Chile), for sharing their time and expertise on this subject.

\section{Declarations}

\section{Conflict of interest None.}

\section{References}

1. Siddighi S, Barker M, Pancholy A, et al. Attitudes and perceptions regarding subspecialty training in female pelvic medicine and reconstructive surgery. Int Urogynecol J. 2008;19:1523-6. https://doi.org/10.1007/s00192-008-0677-9.

2. Gupta A, Kennedy B, Meriwether K, et al. Citation classics: the 100 most cited articles in Urogynecology. Int Urogynecol J. 2020. https://doi.org/10.1007/s00192-019-04021-9.

3. Association of American Medical Colleges (2021) Table C8. Fulltime faculty-appointment status at U.S. medical schools for residents who completed residencies, by specialty and rank. https:// www.aamc.org/data-reports/students-residents/interactive-data/ report-residents/2021/table-c8-full-time-faculty-appointment-status-us-medical-schools-residents Accessed 27th Dec 2021.

Publisher's note Springer Nature remains neutral with regard to jurisdictional claims in published maps and institutional affiliations. 